\title{
A GENERALIZATION OF FUZZY SUBSEMIGROUPS IN SEMIGROUPS
}

\author{
Mee Kwang Kang ${ }^{\mathrm{a}, *}$, Hee Young Ban ${ }^{\mathrm{b}}$ And SAng WoOK Yun ${ }^{\mathrm{c}}$
}

\begin{abstract}
As a generalization of fuzzy subsemigroups, the notion of $\varepsilon$-generalized fuzzy subsemigroups is introduced, and several properties are investigated. A condition for an $\varepsilon$-generalized fuzzy subsemigroup to be a fuzzy subsemigroup is considered. Characterizations of $\varepsilon$-generalized fuzzy subsemigroups are established, and we show that the intersection of two $\varepsilon$-generalized fuzzy subsemigroups is also an $\varepsilon$-generalized fuzzy subsemigroup. A condition for an $\varepsilon$-generalized fuzzy subsemigroup to be $\varepsilon$-fuzzy idempotent is discussed. Using a given $\varepsilon$-generalized fuzzy subsemigroup, a new $\varepsilon$-generalized fuzzy subsemigroup is constructed. Finally, the fuzzy extension of an $\varepsilon$-generalized fuzzy subsemigroup is considered.
\end{abstract}

\section{INTRODUCTION}

The concept of a fuzzy set was first introduced by Zadeh [16] and this concept was adapted by Rosenfeld [14] to define fuzzy subgroups and fuzzy ideals. Based on this pioneering work, Kuroki $[6,7,8,9,10]$ introduced fuzzy semigroups and various kinds of fuzzy ideals in semigroups and characterized certain semigroups using those fuzzy ideals. Since then the literature of various fuzzy algebraic concepts has been growing very rapidly. In the literature, the relationships between the fuzzy sets and semigroups have been considered by many authors (see $[1,2,3,4,5,6,7,8,9,10$, $11,13,15])$.

The aim of this paper is to consider more general form of fuzzy subsemigroups. We define the notion of $\varepsilon$-generalized fuzzy subsemigroups, and investigate several properties. We provide a condition for an $\varepsilon$-generalized fuzzy subsemigroup to be a fuzzy subsemigroup. We establish characterizations of $\varepsilon$-generalized fuzzy subsemigroups. We show that the intersection of two $\varepsilon$-generalized fuzzy subsemigroups is also an $\varepsilon$ generalized fuzzy subsemigroup. We consider a condition for an $\varepsilon$-generalized fuzzy

Received by the editors February 12, 2013. Accepted April 22, 2013.

2010 Mathematics Subject Classification. 20M12, 08A72.

Key words and phrases. $\varepsilon$-generalized fuzzy subsemigroup, $\varepsilon$-product, $\varepsilon$-Cartesian product, fuzzy $\alpha$-translation, $\left(\varepsilon_{1}, \varepsilon_{2}\right)$-fuzzy extension.

${ }^{*}$ Corresponding author. 
subsemigroup to be $\varepsilon$-fuzzy idempotent. Using a given $\varepsilon$-generalized fuzzy subsemigroup, we establish a new $\varepsilon$-generalized fuzzy subsemigroup. Finally, we discuss fuzzy extension of an $\varepsilon$-generalized fuzzy subsemigroup.

\section{Preliminaries}

Let $S$ be a semigroup. Let $A$ and $B$ be subsets of $S$. Then the multiplication of $A$ and $B$ is defined as follows:

$$
A B=\{a b \in S \mid a \in A \text { and } b \in B\} .
$$

Let $S$ be a semigroup. By a subsemigroup of $S$ we mean a nonempty subset $A$ of $S$ such that $A^{2} \subseteq A$. A fuzzy subset $\mu$ of $S$ is called a fuzzy subsemigroup of $S$ if it satisfies:

$$
(\forall x, y \in S)(\mu(x y) \geq \min \{\mu(x), \mu(y)\}) .
$$

Let $\mu$ and $\nu$ be fuzzy subsets of $S$. The product $\mu \circ \nu$ of $\mu$ and $\nu$ is defined by

$$
\mu \circ \nu(x)= \begin{cases}\sup _{x=y z} \min \{\mu(y), \nu(z)\} & \text { if } \exists y, z \in S \text { such that } x=y z, \\ 0 & \text { otherwise. }\end{cases}
$$

We refer the reader to the book [12] for further information regarding fuzzy semigroups.

\section{A Generalization of Fuzzy Subsemigroups}

In what follows, let $\varepsilon \in \mathbb{R}^{+}$where $\mathbb{R}^{+}$is the set of all positive real numbers unless otherwise specified.

Definition 3.1. A fuzzy subset $\mu$ of $S$ is called an $\varepsilon$-generalized fuzzy subsemigroup of $S$ if the following assertions is valid:

$$
(\forall x, y \in S)(\mu(x y) \geq \min \{\mu(x), \mu(y), \varepsilon\}) .
$$

If $\varepsilon \geq 1$, the $\varepsilon$-generalized fuzzy subsemigroup of $S$ is a fuzzy subsemigroup of $S$.

Example 3.2. Let $S$ be a semigroup of four elements $\{a, b, c, d\}$ with the following multiplication table:

\begin{tabular}{c|cccc} 
& $a$ & $b$ & $c$ & $d$ \\
\hline$a$ & $a$ & $a$ & $a$ & $a$ \\
$b$ & $a$ & $a$ & $a$ & $a$ \\
$c$ & $a$ & $a$ & $b$ & $a$ \\
$d$ & $a$ & $a$ & $b$ & $b$
\end{tabular}


Let $\mu$ be a fuzzy subset of $S$ defined by

$$
\mu: S \rightarrow[0,1], x \mapsto\left\{\begin{array}{cl}
0.8 & \text { if } x=a, \\
0.5 & \text { if } x=b, \\
0.7 & \text { if } x=c, \\
0.3 & \text { if } x=d .
\end{array}\right.
$$

Note that $\mu$ is not a fuzzy subsemigroup of $S$. If $\varepsilon \leq 0.5$, then $\mu$ is an $\varepsilon$-generalized fuzzy subsemigroup of $S$. If $0.5<\varepsilon \leq 0.7$, then $\mu$ is not an $\varepsilon$-generalized fuzzy subsemigroup of $S$ since

$$
\mu(c c)=\mu(b)=0.5<\varepsilon=\min \{\mu(c), \mu(c), \varepsilon\} .
$$

Obviously, every fuzzy subsemigroup is an $\varepsilon$-generalized fuzzy subsemigroup for any $\varepsilon$, but the converse is not true in general. For example, Example 3.2 shows that there exists $\varepsilon$ such that $\mu$ is an $\varepsilon$-generalized fuzzy subsemigroup of $S$ which is not a fuzzy subsemigroup of $S$.

Example 3.3. Let $S$ be a semigroup of four elements $\{e, a, b, c\}$ with the following multiplication table:

\begin{tabular}{l|llll} 
& $e$ & $a$ & $b$ & $c$ \\
\hline$e$ & $e$ & $a$ & $b$ & $c$ \\
$a$ & $a$ & $e$ & $b$ & $c$ \\
$b$ & $b$ & $c$ & $b$ & $c$ \\
$c$ & $c$ & $b$ & $b$ & $c$
\end{tabular}

Let $\mu$ be a fuzzy subset of $S$ defined by

$$
\mu: S \rightarrow[0,1], x \mapsto \begin{cases}0.9 & \text { if } x=e, \\ 0.7 & \text { if } x=a, \\ 0.6 & \text { if } x=b, \\ 0.6 & \text { if } x=c .\end{cases}
$$

It is routine to verify that $\mu$ is an $\varepsilon$-generalized fuzzy subsemigroup of $S$ for every $\varepsilon$.

Proposition 3.4. Let $\mu$ be an $\varepsilon$-generalized fuzzy subsemigroup of $S$. If $\varepsilon \geq \mu(x)$ for all $x \in S$, then $\mu$ is a fuzzy subsemigroup of $S$.

Proof. It is straightforward.

Proposition 3.5. Let $\varepsilon_{1}$ and $\varepsilon_{2}$ be any elements of $\mathbb{R}^{+}$. If $\varepsilon_{1} \leq \varepsilon_{2}$, then every $\varepsilon_{2}$-generalized fuzzy subsemigroup is an $\varepsilon_{1}$-generalized fuzzy subsemigroup.

Proof. Straightforward.

The following examples shows that there exist $\varepsilon_{1}, \varepsilon_{2} \in \mathbb{R}^{+}$with $\varepsilon_{1} \leq \varepsilon_{2}$ such that 
any $\varepsilon_{1}$-generalized fuzzy subsemigroup may not be an $\varepsilon_{2}$-generalized fuzzy subsemigroup.

Example 3.6. Let $S=\{e, a, b, c\}$ be a semigroup which is given in Example 3.3 and let $\mu$ be a fuzzy subset of $S$ defined by

$$
\mu: S \rightarrow[0,1], x \mapsto \begin{cases}0.8 & \text { if } x=e, \\ 0.5 & \text { if } x=a, \\ 0.3 & \text { if } x=b, \\ 0.6 & \text { if } x=c .\end{cases}
$$

If we take $\varepsilon_{1} \in[0,0.3]$ and $\varepsilon_{2} \in(0.3,0.5]$, then $\mu$ is an $\varepsilon_{1}$-generalized fuzzy subsemigroup of $S$. Since $\mu(c a)=\mu(b)=0.3<\varepsilon_{2}=\left\{\mu(c), \mu(a), \varepsilon_{2}\right\}, \mu$ is not an $\varepsilon_{2}$-generalized fuzzy subsemigroup of $S$.

Theorem 3.7. Let $\mu$ be a fuzzy subset of $S$. Then $\mu$ is an $\varepsilon$-generalized fuzzy subsemigroup of $S$ if and only if $\mu_{t}$ is a subsemigroup of $S$ for all $t \in[0,1]$ with $t \leq \varepsilon$.

Proof. Assume that $\mu$ is an $\varepsilon$-generalized fuzzy subsemigroup of $S$. Let $x, y \in S$ be such that $x, y \in \mu_{t}$ for all $t \in[0,1]$ with $t \leq \varepsilon$. Then

$$
\mu(x y) \geq \min \{\mu(x), \mu(y), \varepsilon\} \geq \min \{t, \varepsilon\}=t,
$$

and so $x y \in \mu_{t}$. Therefore $\mu_{t}$ is a subsemigroup of $S$ for all $t \in[0,1]$ with $t \leq \varepsilon$.

Conversely, suppose that $\mu_{t}$ is a subsemigroup of $S$ for all $t \in[0,1]$ with $t \leq \varepsilon$. Assume that $\mu$ is not an $\varepsilon$-generalized fuzzy subsemigroup of $S$. Then there exist $a, b \in S$ such that

$$
\mu(a b)<\min \{\mu(a), \mu(b), \varepsilon\} .
$$

Putting $k$ equal to $\min \{\mu(a), \mu(b), \varepsilon\}$, we have $\mu(a b)<k, a, b \in \mu_{k}$ and $k \in[0,1]$ with $k \leq \varepsilon$. It follows that $a b \in \mu_{k}$ which is a contradiction. Hence

$$
\mu(x y) \geq \min \{\mu(x), \mu(y), \varepsilon\}
$$

for all $x, y \in S$. Thus $\mu$ is an $\varepsilon$-generalized fuzzy subsemigroup of $S$.

Let $\mu$ and $\nu$ be fuzzy subsets of $S$. The $\varepsilon$-product $\mu \circ_{\varepsilon} \nu$ of $\mu$ and $\nu$ is defined by

$$
\mu \circ_{\varepsilon} \nu(x)= \begin{cases}\sup _{x=y z} \min \{\mu(y), \nu(z), \varepsilon\} & \text { if } \exists y, z \in S \text { such that } x=y z, \\ 0 & \text { otherwise. }\end{cases}
$$

Theorem 3.8. For a fuzzy subset $\mu$ of $S, \mu$ is an $\varepsilon$-generalized fuzzy subsemigroup of $S$ if and only if $\mu \circ_{\varepsilon} \mu \subseteq \mu$. 
Proof. Assume that $\mu$ is an $\varepsilon$-generalized fuzzy subsemigroup of $S$. Let $x \in S$. If $x \neq y z$ for all $x, y \in S$, then clearly $\mu \circ_{\varepsilon} \mu \subseteq \mu$. If $x=a b$ for some $a, b \in S$, then

$$
\mu \circ_{\varepsilon} \mu(x)=\sup _{x=a b} \min \{\mu(a), \mu(b), \varepsilon\} \leq \sup _{x=a b} \mu(a b)=\mu(x) .
$$

Therefore $\mu \circ_{\varepsilon} \mu \subseteq \mu$.

Conversely, suppose that $\mu \circ_{\varepsilon} \mu \subseteq \mu$. Let $x, y \in S$. Set $a=x y$. Then we have

$$
\mu(x y)=\mu(a) \geq(\mu \circ \varepsilon \mu)(a)=\sup _{a=b c} \min \{\mu(b), \mu(c), \varepsilon\} \geq \min \{\mu(x), \mu(y), \varepsilon\},
$$

and so $\mu$ is an $\varepsilon$-generalized fuzzy subsemigroup of $S$.

If we take $\varepsilon=1$ in Theorem 3.8, then we have the following corollary.

Corollary 3.9 ([12]). For a fuzzy subset $\mu$ of $S, \mu$ is a fuzzy subsemigroup of $S$ if and only if $\mu \circ \mu \subseteq \mu$.

Theorem 3.10. Let $S$ be a semigroup with identity e and let $\mu$ be a fuzzy subset of $S$ such that

$$
(\forall x \in S)(\mu(x) \leq \mu(e) \leq \varepsilon) .
$$

If $\mu$ is an $\varepsilon$-generalized fuzzy subsemigroup of $S$, then it is $\varepsilon$-fuzzy idempotent, that is, $\mu \circ_{\varepsilon} \mu=\mu$.

Proof. Let $x \in S$. Then

$$
\begin{aligned}
\left(\mu \circ_{\varepsilon} \mu\right)(x) & =\sup _{x=y z} \min \{\mu(y), \mu(z), \varepsilon\} \\
& \geq \min \{\mu(e), \mu(x), \varepsilon\}=\mu(x),
\end{aligned}
$$

and so $\mu \circ_{\varepsilon} \mu \subseteq \mu$. It follows from Theorem 3.8 that $\mu \circ_{\varepsilon} \mu=\mu$, that is, $\mu$ is $\varepsilon$-fuzzy idempotent.

Corollary 3.11. Let $S$ be a semigroup with identity e. Then every fuzzy subsemigroup $\mu$ of $S$ is fuzzy idempotent, that is $\mu \circ \mu=\mu$.

Theorem 3.12. For any $\varepsilon_{1}, \varepsilon_{2} \in \mathbb{R}^{+}$, let $\mu$ and $\nu$ be an $\varepsilon_{1}$-generalized fuzzy subsemigroup and an $\varepsilon_{2}$-generalized fuzzy subsemigroup of $S$ respectively. Then $\mu \cap \nu$ is an $\varepsilon$-generalized fuzzy subsemigroup of $S$ where $\varepsilon=\min \left\{\varepsilon_{1}, \varepsilon_{2}\right\}$.

Proof. Let $\varepsilon=\min \left\{\varepsilon_{1}, \varepsilon_{2}\right\}$ and let $x$ and $y$ be any elements of $S$. Then

$$
\begin{aligned}
& (\mu \cap \nu)(x y)=\min \{\mu(x y), \nu(x y)\} \\
& \geq \min \left\{\min \left\{\mu(x), \mu(y), \varepsilon_{1}\right\}, \min \left\{\nu(x), \nu(y), \varepsilon_{2}\right\}\right\} \\
& \geq \min \{\min \{\mu(x), \mu(y), \varepsilon\}, \min \{\nu(x), \nu(y), \varepsilon\}\}
\end{aligned}
$$




$$
\begin{aligned}
& =\min \{\min \{\mu(x), \nu(x)\}, \min \{\mu(y), \nu(y)\}, \varepsilon\} \\
& =\min \{(\mu \cap \nu)(x),(\mu \cap \nu)(y), \varepsilon\} .
\end{aligned}
$$

Therefore $\mu \cap \nu$ is an $\varepsilon$-generalized fuzzy subsemigroup of $S$.

Corollary 3.13. Let $\mu$ and $\nu$ be two $\varepsilon$-generalized fuzzy subsemigroups of $S$. Then $\mu \cap \nu$ is an $\varepsilon$-generalized fuzzy subsemigroup of $S$.

The following example shows that the union of two $\varepsilon$-generalized fuzzy subsemigroups is not an $\varepsilon$-generalized fuzzy subsemigroup.

Example 3.14. Consider the semigroup $S=\{e, a, b, c\}$ which is given in Example 3.3. Let $\mu$ and $\nu$ be fuzzy subsets of $S$ defined by

$$
\mu: S \rightarrow[0,1], x \mapsto \begin{cases}0.6 & \text { if } x \in\{e, a\}, \\ 0.5 & \text { if } x \in\{b, c\}\end{cases}
$$

and

$$
\nu: S \rightarrow[0,1], x \mapsto \begin{cases}0.7 & \text { if } x \in\{e, b\} \\ 0.5 & \text { if } x \in\{a, c\}\end{cases}
$$

respectively. Routine calculations, we know that $\mu$ and $\nu$ are $\varepsilon$-generalized fuzzy subsemigroups of $S$. The union of $\mu$ and $\nu$ is given as follows:

$$
\mu \cup \nu: S \rightarrow[0,1], x \mapsto \begin{cases}0.7 & \text { if } x \in\{e, b\}, \\ 0.6 & \text { if } x=a, \\ 0.5 & \text { if } x=c .\end{cases}
$$

If we put $\varepsilon=0.6$ and take $t \in(0.5,0.6]$, then $(\mu \cup \nu)_{t}=\{e, a, b\}$ is not a subalgebra of $S$. It follows from Theorem 3.7 that $\mu \cup \nu$ is not an $\varepsilon$-generalized fuzzy subsemigroup of $S$.

Theorem 3.15. Let $\mu$ be an $\varepsilon$-generalized fuzzy subsemigroup of $S$. If $k=\sup _{x \in S} \mu(x)$, then the set

$$
S_{\mu}:=\{x \in S \mid \mu(x) \geq \min \{k, \varepsilon\}\}
$$

is a subsemigroup of $S$.

Proof. Let $x, y \in S_{\mu}$. Then $\mu(x) \geq \min \{k, \varepsilon\}$ and $\mu(y) \geq \min \{k, \varepsilon\}$. If follows from (3.1) that

$$
\mu(x y) \geq \min \{\mu(x), \mu(y), \varepsilon\} \geq \min \{k, \varepsilon\} .
$$

Hence $x y \in S_{\mu}$, and thus $S_{\mu}$ is a subsemigroup of $S$. 
Let $S$ and $T$ be semigroups. For a map $f: S \rightarrow T$ and a fuzzy subset $\mu$ of $T$, define a fuzzy subset $\mu_{f}^{\varepsilon}$ of $S$ by

$$
\mu_{f}^{\varepsilon}: S \rightarrow[0,1], x \mapsto \min \{\mu(f(x)), \varepsilon\} .
$$

Theorem 3.16. Let $f: S \rightarrow T$ be a homomorphism of semigroups. If $\mu$ is an $\varepsilon$ generalized fuzzy subsemigroup of $T$, then $\mu_{f}^{\varepsilon}$ is an $\varepsilon$-generalized fuzzy subsemigroup of $S$.

Proof. Let $x, y \in S$. Then

$$
\begin{aligned}
\mu_{f}^{\varepsilon}(x y) & =\min \{\mu(f(x y)), \varepsilon\}=\min \{\mu(f(x) f(y)), \varepsilon\} \\
& \geq \min \{\min \{\mu(f(x)), \mu(f(y)), \varepsilon\}, \varepsilon\} \\
& =\min \{\min \{\mu(f(x)), \varepsilon\}, \min \{\mu(f(y)), \varepsilon\}, \varepsilon\} \\
& =\min \left\{\mu_{f}^{\varepsilon}(x), \mu_{f}^{\varepsilon}(y), \varepsilon\right\} .
\end{aligned}
$$

Hence $\mu_{f}^{\varepsilon}$ is an $\varepsilon$-generalized fuzzy subsemigroup of $S$.

For two $\varepsilon$-generalized fuzzy subsemigroups $\mu$ and $\nu$ of $S$, we define the $\varepsilon$-Cartesian product of $\mu$ and $\nu$ as follows:

$$
\mu \times_{\varepsilon} \nu: S \times S \rightarrow[0,1],(x, y) \mapsto \min \{\mu(x), \nu(y), \varepsilon\} .
$$

Theorem 3.17. If $\mu$ and $\nu$ are $\varepsilon$-generalized fuzzy subsemigroups of $S$, then the $\varepsilon$-Cartesian product of $\mu$ and $\nu$ is an $\varepsilon$-generalized fuzzy subsemigroup of $S \times S$.

Proof. Let $(x, y),(a, b) \in S \times S$. Then

$$
\begin{aligned}
&\left(\mu \times_{\varepsilon} \nu\right)((x, y)(a, b))=\left(\mu \times_{\varepsilon} \nu\right)((x a, y b))=\min \{\mu(x a), \nu(y b), \varepsilon\} \\
& \quad \geq \min \{\min \{\mu(x), \mu(a), \varepsilon\}, \min \{\nu(y), \nu(b), \varepsilon\}, \varepsilon\} \\
& \quad=\min \{\min \{\mu(x), \nu(y), \varepsilon\}, \min \{\mu(a), \nu(b), \varepsilon\}, \varepsilon\} \\
& \quad=\min \left\{\left(\mu \times_{\varepsilon} \nu\right)(x, y),\left(\mu \times_{\varepsilon} \nu\right)(a, b), \varepsilon\right\},
\end{aligned}
$$

and so $\mu \times_{\varepsilon} \nu$ is an $\varepsilon$-generalized fuzzy subsemigroup of $S \times S$.

For a fuzzy subset $\mu$ of $S$, we define a new fuzzy subset $\mu^{*}$ of $S$ as follows:

$$
\mu^{*}: S \rightarrow[0,1], x \mapsto \mu(x)+1-k
$$

where $k=\sup _{x \in S} \mu(x)$.

Theorem 3.18. If $\mu$ is an $\varepsilon$-generalized fuzzy subsemigroup of $S$, then so is $\mu^{*}$. 
Proof. Let $x, y \in S$. Note that $\varepsilon+1-k \geq \varepsilon$. Hence

$$
\begin{aligned}
\mu^{*}(x y) & =\mu(x y)+1-k \geq \min \{\mu(x), \mu(y), \varepsilon\}+1-k \\
& =\min \{\mu(x)+1-k, \mu(y)+1-k, \varepsilon+1-k\} \\
& =\min \left\{\mu^{*}(x), \mu^{*}(y), \varepsilon\right\},
\end{aligned}
$$

and so $\mu^{*}$ is an $\varepsilon$-generalized fuzzy subsemigroup of $S$.

For any fuzzy subset $\mu$ of $S$, we denote $\top:=1-\sup \{\mu(x) \mid x \in S\}$. For any $\alpha \in[0, \top]$, a mapping $\mu_{\alpha}^{t}: S \rightarrow[0,1]$ is called a fuzzy $\alpha$-translation of $\mu$ if it satisfies:

$$
(\forall x \in S)\left(\mu_{\alpha}^{t}(x)=\mu(x)+\alpha\right) .
$$

Theorem 3.19. Let $\mu$ be an $\varepsilon$-generalized fuzzy subsemigroup of $S$ and let $\alpha \in[0, \top]$ with $\alpha+\varepsilon \leq 1$. Then the fuzzy $\alpha$-translation $\mu_{\alpha}^{t}$ of $\mu$ is an $\alpha+\varepsilon$-generalized fuzzy subsemigroup of $S$.

Proof. Let $x, y \in S$. Then

$$
\begin{aligned}
\mu_{\alpha}^{t}(x y) & =\mu(x y)+\alpha \geq \min \{\mu(x), \mu(y), \varepsilon\}+\alpha \\
& =\min \{\mu(x)+\alpha, \mu(y)+\alpha, \alpha+\varepsilon\} \\
& =\min \left\{\mu_{\alpha}^{t}(x), \mu_{\alpha}^{t}(y), \alpha+\varepsilon\right\} .
\end{aligned}
$$

Therefore $\mu_{\alpha}^{t}$ is an $\alpha+\varepsilon$-generalized fuzzy subsemigroup of $S$.

Theorem 3.20. Let $\mu$ be a fuzzy subset of $S$ such that the fuzzy $\alpha$-translation $\mu_{\alpha}^{t}$ of $\mu$ is an $\alpha+\varepsilon$-generalized fuzzy subsemigroup of $S$ for some $\alpha \in[0, \top]$ with $\alpha+\varepsilon \leq 1$. Then $\mu$ is an $\varepsilon$-generalized fuzzy subsemigroup of $S$.

Proof. Assume that $\mu_{\alpha}^{t}$ is an $\alpha+\varepsilon$-generalized fuzzy subsemigroup of $S$ for some $\alpha \in[0, \top]$ with $\alpha+\varepsilon \leq 1$. Let $x, y \in S$. Then

$$
\begin{aligned}
\mu(x y)+\alpha & =\mu_{\alpha}^{t}(x y) \geq \min \left\{\mu_{\alpha}^{t}(x), \mu_{\alpha}^{t}(y), \alpha+\varepsilon\right\} \\
& =\min \{\mu(x)+\alpha, \mu(y)+\alpha, \alpha+\varepsilon\} \\
& =\min \{\mu(x), \mu(y), \varepsilon\}+\alpha,
\end{aligned}
$$

and so $\mu(x y) \geq \min \{\mu(x), \mu(y), \varepsilon\}$. Consequently, $\mu$ is an $\varepsilon$-generalized fuzzy subsemigroup of $S$.

Let $\mu_{1}$ and $\mu_{2}$ be fuzzy subsets of $S$ and $\varepsilon_{1}, \varepsilon_{2} \in \mathbb{R}^{+}$. We say that $\mu_{2}$ is an $\left(\varepsilon_{1}, \varepsilon_{2}\right)$-fuzzy extension of $\mu_{1}$ if

(1) $(\forall x \in S)\left(\mu_{1}(x) \leq \mu_{2}(x)\right)$, 
(2) If $\mu_{1}$ is an $\varepsilon_{1}$-generalized fuzzy subsemigroup of $S$, then $\mu_{2}$ is an $\varepsilon_{2}$-generalized fuzzy subsemigroup of $S$.

Let $S$ be the semigroup and $\mu$ be the fuzzy subset of $S$ as in Example 3.6. Define a fuzzy subset $\nu$ of $S$ as follows:

$$
\nu: S \rightarrow[0,1], x \mapsto \begin{cases}0.9 & \text { if } x=e, \\ 0.7 & \text { if } x=a, \\ 0.4 & \text { if } x=b, \\ 0.8 & \text { if } x=c .\end{cases}
$$

Then $\mu(x) \leq \nu(x)$ for all $x \in S$ and note that $\mu$ is an $\varepsilon_{1}$-generalized fuzzy subsemigroup of $S$ with $\varepsilon_{1}=0.2$ (see Example 3.6). If we take $\varepsilon_{2} \in(0.2,0.3$ ], then $\nu$ is an $\varepsilon_{2}$-generalized fuzzy subsemigroup of $S$. Hence $\nu$ is an $\left(\varepsilon_{1}, \varepsilon_{2}\right)$-fuzzy extension of $\mu$. But, if we take $\varepsilon_{3} \in(0.4,0.7]$, then $\nu$ is not an $\varepsilon_{2}$-generalized fuzzy subsemigroup of $S$ since $\nu_{t}=\{e, a, c\}$ is not a subsemigroup of $S$ for $t \in\left(0.4, \varepsilon_{3}\right]$. Therefore $\nu$ is not an $\left(\varepsilon_{1}, \varepsilon_{3}\right)$-fuzzy extension of $\mu$.

By means of the definition of fuzzy $\alpha$-translation, we know that $\mu_{\alpha}^{t}(x) \geq \mu(x)$ for all $x \in S$. Hence the following theorem is implied by Theorem 3.19.

Theorem 3.21. Let $\mu$ be an $\varepsilon$-generalized fuzzy subsemigroup of $S$ and $\alpha \in[0, \top]$ with $\alpha+\varepsilon \leq 1$. Then the fuzzy $\alpha$-translation $\mu_{\alpha}^{t}$ of $\mu$ is an $(\varepsilon, \alpha+\varepsilon)$-fuzzy extension of $\mu$.

The following example illustrates Theorem 3.21.

Example 3.22. Let $S$ be a semigroup of four elements $\{e, a, b, c\}$ with the following multiplication table:

\begin{tabular}{c|cccc} 
& $e$ & $a$ & $b$ & $c$ \\
\hline$e$ & $e$ & $a$ & $b$ & $c$ \\
$a$ & $a$ & $e$ & $b$ & $c$ \\
$b$ & $b$ & $c$ & $b$ & $c$ \\
$c$ & $c$ & $b$ & $b$ & $c$
\end{tabular}

Let $\mu$ be a fuzzy subset of $S$ defined by

$$
\mu: S \rightarrow[0,1], x \mapsto \begin{cases}0.85 & \text { if } x=e, \\ 0.8 & \text { if } x=a, \\ 0.6 & \text { if } x=b, \\ 0.55 & \text { if } x=c .\end{cases}
$$


Then $\mu$ is an $\varepsilon$-generalized fuzzy subsemigroup of $S$ for $\varepsilon=0.4$. For any $\alpha=0.02 \epsilon$ $[0,0.15]$, the fuzzy $\alpha$-translation $\mu_{\alpha}^{t}$ of $\mu$ is given as follows:

$$
\mu_{\alpha}^{t}: S \rightarrow[0,1], x \mapsto \begin{cases}0.87 & \text { if } x=e, \\ 0.82 & \text { if } x=a, \\ 0.62 & \text { if } x=b, \\ 0.57 & \text { if } x=c,\end{cases}
$$

which is an $(\varepsilon, \alpha+\varepsilon)$-fuzzy extension of $\mu$.

\section{REFERENCES}

1. K.A. Dib \& N. Galhum: Fuzzy ideals and fuzzy bi-ideals in fuzzy semigroups. Fuzzy Sets and Systems 92 (1997), 103-111.

2. Y.B. Jun \& S.Z. Song: Generalized fuzzy interior-ideals in semigroups. Inform. Sci. 176 (2006), no. 20, 3079-3093.

3. S.M. Hong, Y.B. Jun \& J. Meng: Fuzzy interior-ideals in semigroups. Indian J. Pure Appl. Math. 26 (1995), 859-863.

4. O. Kazanci \& S. Yamak: Generalized fuzzy bi-ideals of semigroups. Soft Computing 12 (2008), 1119-1124.

5. __ : Fuzzy ideals and semiprime fuzzy ideals in semigroups. Inform. Sci. 179 (2009), 3720-3731.

6. N. Kuroki: Fuzzy hi-ideals in semigroups. Comment. Math., Univ. St. Paul. 28 (1979), 17-21.

7. _ : On fuzzy ideals and fuzzy bi-ideals in semigroups. Fazzy Sets and Systems $\mathbf{5}$ (1981), 203-215

8. _ : Fuzzy semiprime ideals in semigroups. Fazzy Sets and Systems 8 (1982), 71-79.

9. __ : On fuzzy semigroups. Inform. Sci. 53 (1991), 203-236.

10. ___ Fuzzy semiprime quasi-ideals in semigroups. Inform. Sci. 75 (1993), no. 3, 201-211.

11. Z.W. Mo \& X.P. Wang: On pointwise depiction of fuzzy regularity of semigroups. Inform. Sci. 74 (1993), 265-274.

12. J.N. Mordeson, D.S. Malik \& N. Kuroki: Fuzzy semigroups. Springer-Verlag Berlin Heidelberg New York, 2003.

13. X. Pan \& Y. Xu: Lattice implication ordered semigroups. Inform. Sci. 178 (2008), no. $2,403-413$.

14. A. Rosenfeld: Fuzzy groups. J. Math. Anal. Appl. 35 (1971), 512-517.

15. X.Y. Xie: Fuzzy ideals in semigroups. J. Fuzzy Math. 7 (1999), no. 2, 357-365.

16. L.A. Zadeh: Fuzzy sets. Inform. Control 8 (1965), 338-353. 
${ }^{a}$ Department of Mathematics, Dongeui University, Busan 614-714, Korea

Email address: mee@deu.ac.kr

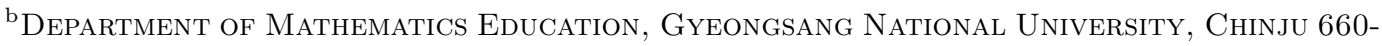
701, KOREA

Email address: ban2329@naver.com

${ }^{\text {c}}$ Department of Mathematics Education, Gyeongsang National University, Chinju 660701, Korea

Email address: yswig@hanmail.net 